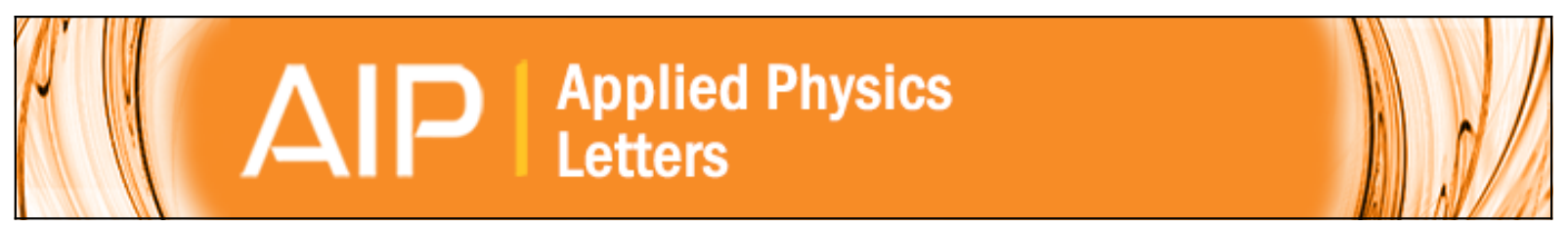

Polarization-resolved extinction and scattering cross-sections of individual gold nanoparticles measured by wide-field microscopy on a large ensemble

Lukas M. Payne, Wolfgang Langbein, and Paola Borri

Citation: Applied Physics Letters 102, 131107 (2013); doi: 10.1063/1.4800564

View online: http://dx.doi.org/10.1063/1.4800564

View Table of Contents: http://scitation.aip.org/content/aip/journal/apl/102/13?ver=pdfcov

Published by the AIP Publishing

$\underset{\substack{\text { Publoning } \\ \text { PIP }}}{A}$ Re-register for Table of Content Alerts 


\title{
Polarization-resolved extinction and scattering cross-sections of individual gold nanoparticles measured by wide-field microscopy on a large ensemble
}

\author{
Lukas M. Payne, ${ }^{1}$ Wolfgang Langbein, ${ }^{2}$ and Paola Borri ${ }^{1, \text { a) }}$ \\ ${ }^{1}$ School of Biosciences, Cardiff University, Cardiff CF24 3AA, United Kingdom \\ ${ }^{2}$ School of Physics and Astronomy, Cardiff University, The Parade, Cardiff CF24 3AA, United Kingdom
}

(Received 29 January 2013; accepted 25 March 2013; published online 4 April 2013)

\begin{abstract}
We report a simple, rapid, and quantitative wide-field technique to measure the optical extinction $\sigma_{\text {ext }}$ and scattering $\sigma_{\text {sca }}$ cross-section of single nanoparticles using wide-field microscopy enabling simultaneous acquisition of hundreds of nanoparticles for statistical analysis. As a proof-of-principle, we measured gold nanoparticles of $40 \mathrm{~nm}$ and $100 \mathrm{~nm}$ diameter and found mean values and standard deviations of $\sigma_{\text {ext }}$ and $\sigma_{\text {sca }}$ consistent with the literature. Switching from unpolarized to linearly polarized excitation, we measured $\sigma_{\text {ext }}$ as a function of the polarization direction and characterized the nanoparticle asphericity. The method can be implemented cost-effectively on any conventional wide-field microscope and is applicable to any nanoparticles. (C) 2013 American Institute of Physics. [http://dx.doi.org/10.1063/1.4800564]
\end{abstract}

Metallic nanoparticles (NPs) exhibit morphologydependent localized surface plasmon resonances (LSPRs), which couple to propagating light and manifest as an increased particle polarisability at the LSPR frequency. Besides fundamental interest, these local optical resonances can be exploited to image metallic NPs with high sensitivity and to probe nanoscale regions in the NP vicinity via the local field enhancement effect, with possible applications ranging from sub-wavelength optical devices, ${ }^{1}$ catalysis ${ }^{2}$ and photovoltaics $^{3}$ to biomedical imaging ${ }^{4,5}$ and sensing. ${ }^{6-8}$

Important physical quantities characterizing the linear optical properties of a NP are the absorption cross-section $\sigma_{\mathrm{abs}}$, the scattering cross-section $\sigma_{\text {sca, }}$ and the resulting extinction crosssection $\sigma_{\text {ext }}=\sigma_{\text {abs }}+\sigma_{\text {sca }}$. Beyond traditional ensemble average measurements, a number of approaches have been developed recently to measure $\sigma_{\text {ext }}$ at the single particle level, showing that the optical properties of individual NPs can significantly differ from the ensemble average owing to inhomogeneities in NP size and shape. It is, therefore, particularly important to develop a technique that is able to rapidly quantify the cross-sections at the single NP level and to perform a statistical analysis over many NPs, providing a relevant sample characterization. Quantitative values of $\sigma_{\text {ext }}, \sigma_{\text {abs, }}$ and/or $\sigma_{\text {sca }}$ of single NPs have been reported using dark-field micro-spectroscopy, ${ }^{9}$ photothermal imaging, ${ }^{10}$ and spatial modulation micro-spectroscopy. ${ }^{11}$ However, in order to provide cross-section values in absolute units, dark-field micro-spectroscopy and photothermal imaging require a calibration reference (e.g., by comparison with $\sigma_{\text {ext }}$ known from theory), while spatial modulation microspectroscopy needs a precise measurement of the beam profile at the sample. Moreover, photothermal imaging and spatial modulation micro-spectroscopy are beam-scanning techniques, therefore, costly and less amenable to the rapid characterization of a large number of NPs compared to wide-field techniques. Additionally, they are modulation-based which requires specialized equipments such as acousto-optical modulators and lock-in detection.

a)Electronic address: BorriP@cardiff.ac.uk
In this work, we report a simple and quantitative widefield technique to measure $\sigma_{\text {ext }}$ and $\sigma_{\text {sca }}$ on single NPs using conventional bright and dark-field microscopy without the need for calibration standards and with a field of view enabling simultaneous acquisition of hundreds of NPs.

The experimental set-up consists of an inverted microscope (Nikon Ti-U) equipped with a white-light illumination (halogen lamp $100 \mathrm{~W}$ with Nikon neutral color balance filter), a oil condenser of 1.4 numerical aperture (NA) with a removable homebuilt dark-field illumination of 1.1-1.4 NA, a $40 \times 0.95$ NA dry objective, a $1.5 \times$ intermediate magnification, and a Canon EOS $40 \mathrm{D}$ color camera attached to the left port of the microscope. Images were taken in Canon 14-bit RAW format with 10.1 megapixel resolution. The RAW images were converted using the DCRAW plugin in IMAGEJ, providing 16 bit RGB images with a linear response to intensity and no color balancing. The investigated samples were gold NPs (GNPs) of nominal $40 \mathrm{~nm}$ and $100 \mathrm{~nm}$ diameter (BBInternational) covalently bound onto a glass coverslip functionalised with (3-mercapto) triethoxysilane, covered in silicone oil (refractive index $n=1.518$ ) and sealed with a glass slide.

Dark-field microscopy was performed initially to confirm the presence of metallic NPs appearing as colored scatterers, distinguishable from the white scattering of other debris or glass roughness. The color camera enables a coarse spectroscopic detection separating the three wavelength ranges of red (R) 570-650 nm, green (G) 480-580 nm, and blue (B) 420-510 nm. Once a suitable region was located and focussed, a dark-field image was taken. Subsequent bright-field microscopy was performed by removing the dark field ring and adjusting the condenser numerical aperture $\mathrm{NA}_{c}$ to match the objective NA. To quantitatively measure the extinction cross-sections, two bright-field transmission images were taken, one with the NPs in the objective focus and the second one out-of-focus, moving the objective by approximately $d=15 \mu \mathrm{m}$ axially away from the sample. Background images were taken for blocked illumination. To achieve the lowest shot noise, the lowest camera sensitivity was used (100 ISO), for which the full-well capacity of the 
pixels of about $N_{\mathrm{fw}}=4 \times 10^{4}$ electrons occurs at $70 \%$ of the digitizer range (3.4 electrons/count). The exposure time in the order of $10 \mathrm{~ms}$ was chosen to reach $N_{\mathrm{fw}}$. Averaging over $N_{\mathrm{a}}=36$ acquisitions was performed for each image set.

Let us call the background-subtracted transmitted intensity of the bright-field image with NPs in focus $I_{\mathrm{f}}$ and the defocused intensity $I_{\mathrm{d}}$. In the defocused image, a NP distributes its effect over a radius of about $r_{\mathrm{d}}=\mathrm{NA} d$ making $I_{\mathrm{d}}$ similar to the intensity $I_{\mathrm{f}}$ in the absence of the NP. The extinction cross-section of a NP located within the area $A_{\mathrm{i}}$ in the image can then be expressed as $\sigma_{\text {ext }}=\int_{A_{\mathrm{i}}} \Delta d A$ with the relative extinction $\Delta=\left(I_{\mathrm{d}}-I_{\mathrm{f}}\right) / I_{\mathrm{d}}$. An example of a full color dark-field image and the corresponding $\Delta$ image for gold NPs of $40 \mathrm{~nm}$ diameter is shown in Fig. 1. To account for the slight mismatch between $I_{\mathrm{d}}$ and $I_{\mathrm{f}}$ without NP due to the defocusing, drift of the illumination intensity, and the residual influence of the NP, we determine a local background extinction $\Delta_{\mathrm{b}}=A_{\mathrm{b}}{ }^{-1} \int_{A_{\mathrm{b}}} \Delta d A$ in the area $A_{\mathrm{b}}$ between the radius $r_{\mathrm{i}}$ and $2 r_{\mathrm{i}}$, as sketched in Fig. 1(d), yielding the background-corrected $\sigma_{\text {ext }}=\int_{A_{\mathrm{i}}}\left(\Delta-\Delta_{\mathrm{b}}\right) d A$. The correction area $A_{\mathrm{b}}$ is within the defocused image of the NP, i.e., $2 r_{\mathrm{i}}<r_{\mathrm{d}}$, ensuring a homogeneous influence of the NP over $A_{\mathrm{i}}$ and $A_{\mathrm{b}}$. The dependence of the measured $\sigma_{\mathrm{ext}}$ on $r_{\mathrm{i}}$ is shown in the inset of Fig. 1(b) for the $G$ channel, using a constant $\Delta_{\mathrm{b}}$ from $r_{\mathrm{i}}=1.5 \mu \mathrm{m}$. A saturation of $\sigma_{\mathrm{ext}}$ is observed for $r_{\mathrm{i}}>800 \mathrm{~nm} \sim 3 \lambda /(2 \mathrm{NA})$, approximately at the second airy ring of the objective point-spread function. This behavior can be qualitatively understood considering that $\Delta$ is the result of the interference between the scattered field of the NP and the illumination field. For a spatially coherent illumination, this interference would lead to fringes in $\sigma_{\text {ext }}$ decaying as $1 / \sqrt{r}$. However, the short spatial coherence length $d_{\mathrm{c}} \sim \lambda / \mathrm{NA}_{\mathrm{c}}$ of the illumination is suppressing these fringes for $r>d_{\mathrm{c}}$.

In order to determine $\sigma_{\text {ext }}$ of many particles from an extinction image, we developed an image analysis programme written in IMAGEJ macro language. We split the raw images into RGB channels, subtract the background, average

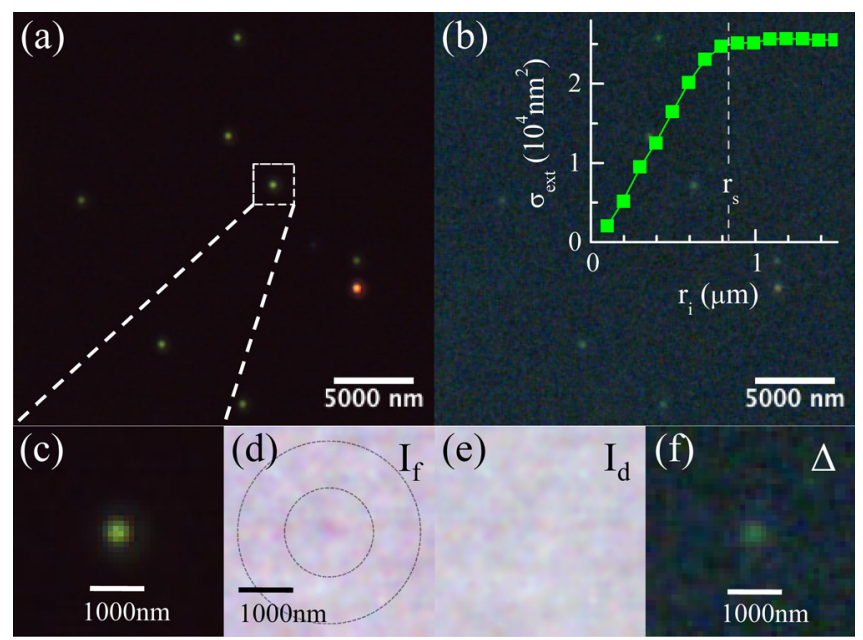

FIG. 1. (a) Full color (FC) dark-field image of $40 \mathrm{~nm}$ diameter gold NPs. (b) Corresponding FC extinction image $\Delta$ from 0 (black) to 0.043 (white). (c) Zoom of dark-field image. Corresponding zoom of the FC bright-field transmission with NP in focus $I_{\mathrm{f}}(\mathrm{d})$ and out-of-focus $I_{\mathrm{d}}$ (e). (f) Zoom of FC extinction image. the multiple acquisitions of $I_{\mathrm{f}}$ and $I_{\mathrm{d}}$, and calculate $\Delta$. We then determine the particle locations as the maxima of $\Delta$ with values in a range adjusted to reject noise and large aggregates. For each maximum, we choose $A_{\mathrm{i}}$ given by a centered disk of radius $r_{\mathrm{i}}=3 \lambda /(2 \mathrm{NA})=837 \mathrm{~nm}$ and calculate $\sigma_{\text {ext }}$.

We select individual NPs using their extinction color, retaining NPs with $\sigma_{\text {ext }}$ largest in the color channel corresponding to the expected plasmon resonance, i.e., green (red) for $40 \mathrm{~nm}(100 \mathrm{~nm})$ spherical GNPs having a LSPR at $540 \mathrm{~nm}(590 \mathrm{~nm})$ in a surrounding medium of 1.5 refractive index. NPs which likely correspond to aggregates, debris, or largely non-spherical GNPs are excluded in this way. The statistical results over 104 individual GNPs of nominally $40 \mathrm{~nm}$ diameter are summarised in Fig. 2. The distribution of $\sigma_{\text {ext }}$ in the $\mathrm{G}$ channel corresponding to the plasmon resonance has a mean of $\bar{\sigma}_{\text {ext }}=4000 \mathrm{~nm}^{2}$, which is consistent with experimental and theoretical values found in the literature. ${ }^{11-14}$ The standard deviation $\hat{\sigma}_{\text {total }}=1300 \mathrm{~nm}^{2}$ of $\sigma_{\text {ext }}$ contains a part $\hat{\sigma}_{\text {noise }}$ due to measurement noise. This part is determined from the distribution of $\sigma_{\text {ext }}$ in image regions not containing NPs, which has a zero mean and a standard deviation $\hat{\sigma}_{\text {noise }}$. We find $\hat{\sigma}_{\text {noise }}=590 \mathrm{~nm}^{2}$ for the $\mathrm{G}$ channel. The standard deviation $\hat{\sigma}_{\text {ext }}$ arising from the NPs is accordingly determined by $\hat{\sigma}_{\text {ext }}^{2}=\hat{\sigma}_{\text {total }}^{2}-\hat{\sigma}_{\text {noise }}^{2}$, resulting in $\hat{\sigma}_{\text {ext }}=960 \mathrm{~nm}^{2}$ for the $\mathrm{G}$ channel. $\hat{\sigma}_{\text {ext }}$ can be attributed to a size distribution of the GNPs as follows. The scaling of $\sigma_{\text {ext }} \propto R^{\gamma}$ for spherical particles of radius $R$ is known from Mie theory. ${ }^{15}$ In the dipole approximation, $\gamma \approx 3$ for small particles, where the extinction is dominated by absorption and increases towards $\gamma=6$ for larger particles, where the extinction is dominated by scattering. We found $\gamma \approx 3$ for $40 \mathrm{~nm}$ diameter at $532 \mathrm{~nm}$ wavelength using calculated absorption and scattering cross-sections. ${ }^{12}$ This scaling allows

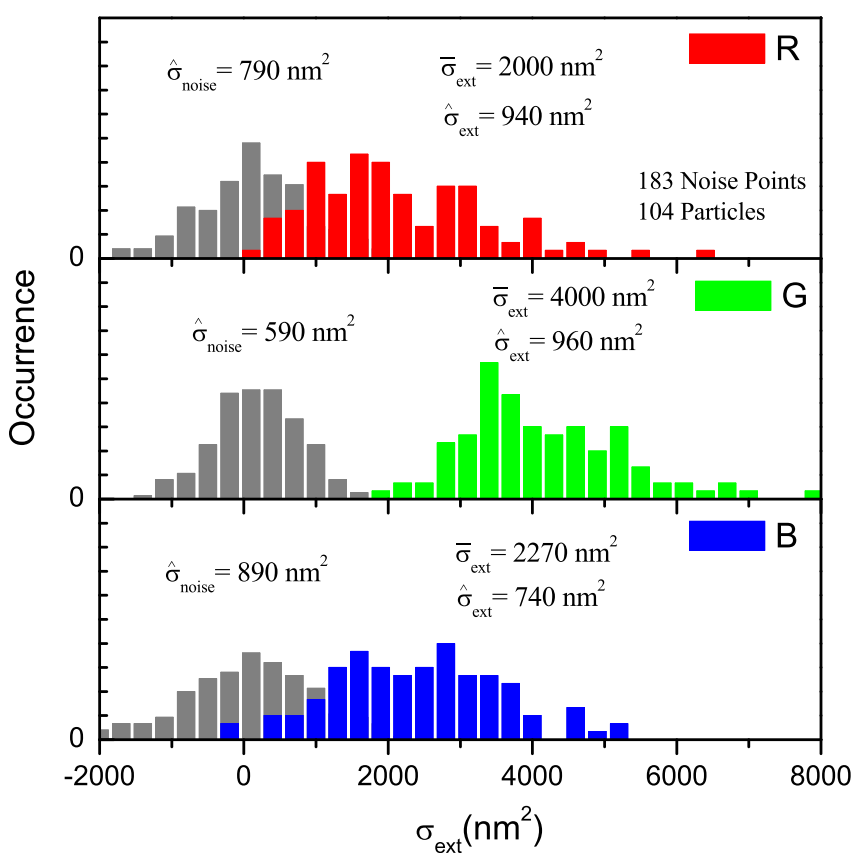

FIG. 2. Distributions of $\sigma_{\text {ext }}$ for 104 GNPs of nominal $40 \mathrm{~nm}$ diameter in the $\mathrm{R}, \mathrm{G}$, and B color channels as indicated. The grey histograms show the noise distribution obtained by measuring $\sigma_{\text {ext }}$ in 183 randomly selected regions without NPs. 
us to estimate the relative standard deviation of the radius $\delta R / R=\hat{\sigma}_{\text {ext }} /\left(\bar{\sigma}_{\text {ext }} \gamma\right) \approx 0.08$. The manufacturer specifies $\delta R / R<0.08$ for $40 \mathrm{~nm}$ and $100 \mathrm{~nm}$ particles determined by electron microscopy. Thus, $\hat{\sigma}_{\text {ext }}$ is on the upper limit of what expected from the size distribution of spherical particles in a constant dielectric environment. It has been shown in the literature that additional factors influencing $\hat{\sigma}_{\text {ext }}$ might be the NP non perfect sphericity, ${ }^{10}$ as well as fluctuations in the local dielectric environment and the electron-surface scattering damping parameter. ${ }^{11}$ Measurements of $\sigma_{\text {ext }}$ for $100 \mathrm{~nm}$ GNPs (not shown) in the red channel yield $\bar{\sigma}_{\text {ext }}=41000 \mathrm{~nm}^{2}$ and $\hat{\sigma}_{\text {ext }}=5847 \mathrm{~nm}^{2}$, resulting in $\delta R / R=0.032$ with $\gamma=4.5$. These values are consistent with the literature ${ }^{11-14}$ for spherical $100 \mathrm{~nm}$ GNPs and meet the manufacturer's size specifications.

Using the scattered intensity $I_{\mathrm{df}}$ measured in dark-field microscopy integrated over the same spatial area $A_{\mathrm{i}}$ as $\sigma_{\text {ext }}$, we obtain the detected power scattered by the NP which is proportional to the scattering cross-section $\sigma_{\text {sca. }}$. Normalizing the scattered intensity to $I_{\mathrm{d}}$, we can write $\sigma_{\text {sca }}=\eta \int_{A_{\mathrm{i}}} I_{\mathrm{df}} d A / I_{\mathrm{d}}$ with the constant $\eta$ determined only by the condenser $\mathrm{NA}_{\mathrm{c}}$ ranges in bright field and dark field and the objective NA. If $\eta$ is known, $\sigma_{\text {sca }}$ can be quantified in absolute units. We determined $\eta$ as follows. We compared $\sigma_{\text {sca }}$ with $\sigma_{\text {ext }}$ on each NP of the ensemble, as shown in Fig. 3. GNPs with $\sigma_{\text {ext }}$ well below a certain cut-off value $\left(\sigma_{\mathrm{c}}\right)$ are dominated by absorption with cross-section $\sigma_{\mathrm{abs}}$ scaling like $R^{3}$. Since $\sigma_{\mathrm{sca}}$ is proportional to $R^{6}$ in this regime, we expect $\sigma_{\text {sca }} \sigma_{\mathrm{c}}=\sigma_{\text {ext }}^{2}$. Conversely, scattering dominates for larger particles such that $\sigma_{\text {ext }} \simeq \sigma_{\text {sca }}$. This trend is indeed observed in Fig. 3, and a fit of the interpolation $1 / \sigma_{\text {sca }}=\sigma_{\mathrm{c}} / \sigma_{\text {ext }}^{2}+1 / \sigma_{\text {ext }}$ allows us to infer $\sigma_{\mathrm{c}}=34000 \mathrm{~nm}^{2}$ and $\eta=26$, both with about $10 \%$ error. The resulting $\sigma_{\text {sca }}$ of the GNPs is consistent with the

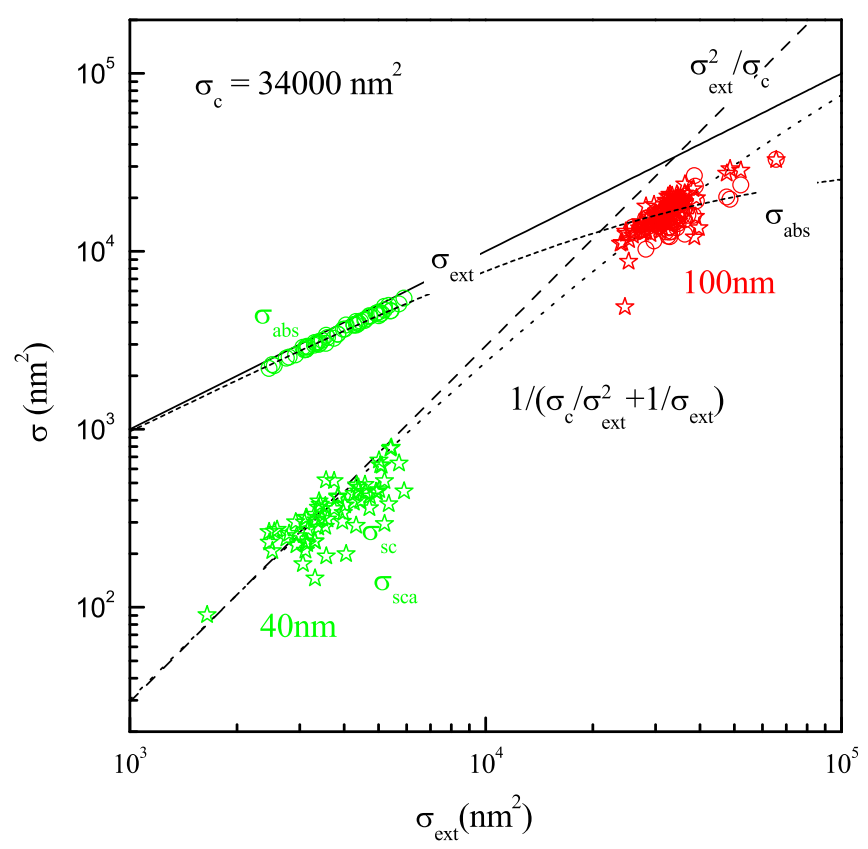

FIG. 3. Scattering cross-section $\sigma_{\text {sca }}$ (stars) measured in dark-field images versus $\sigma_{\text {ext }}$ measured in extinction images for $40 \mathrm{~nm}$ GNPs and $100 \mathrm{~nm}$ GNPs in the green channel. The deduced absorption cross sections $\sigma_{\mathrm{abs}}$ are given as circles. The dotted line gives the fitted scaling as labeled. The limiting behaviour for small $\sigma_{\text {ext }}$ (dashed line) and large $\sigma_{\text {ext }}$ (solid line), and the expected absorption cross-section (short-dashed) are also shown. literature. ${ }^{14}$ Furthermore, we can deduce the absorption cross-section $\sigma_{\mathrm{abs}}=\sigma_{\mathrm{ext}}-\sigma_{\text {sca }}$, which is also shown in Fig. 3. Using the calibrated $\sigma_{\text {sca }}$, we find a detection limit for $\sigma_{\text {sca }}$ of about $100 \mathrm{~nm}^{2}$ limited by the background scattering contributing to $I_{\mathrm{df}}$ in our samples. Increasing the exposure time allows in principle to detect $\sigma_{\text {sca }}<1 \mathrm{~nm}^{2}$ considering the camera dark noise. For the dark-field data shown in Fig. 3 , we used exposure times in the order of $5 \mathrm{~s}$.

While the detection limit for $\sigma_{\text {sca }}$ is given by the sample background scattering, the detection limit for $\sigma_{\text {ext }}$ is given by the shot noise in the measured transmitted intensity. The relative shot noise is given by $1 / \sqrt{N_{\text {ph }}}$ with the detected number of photons $N_{\mathrm{ph}}$, which is determined by the number of acquisitions $N_{\mathrm{a}}$, the full-well capacity $N_{\mathrm{fw}}$ of the camera pixels, the number of pixels $N_{\mathrm{px}}$ in the area $A_{\mathrm{i}}$, and the fraction $\nu$ of pixels used for the color channel (for the Bayer color filter of our camera $\nu=1 / 2$ for $\mathrm{G}$ and $\nu=1 / 4$ for $\mathrm{R}, \mathrm{B}$ ), yielding $\hat{\sigma}_{\text {noise }}=A_{\mathrm{i}} / \sqrt{N_{\mathrm{a}} N_{\mathrm{fw}} \nu N_{\mathrm{px}}}$. With the pixel size $d_{\mathrm{px}}$, the area $A_{\mathrm{i}}=\pi r_{\mathrm{i}}^{2}$ with $r_{\mathrm{i}}=3 \lambda /(2 \mathrm{NA})$, and the magnification $M$ onto the camera, we find

$$
\hat{\sigma}_{\text {noise }}=\frac{3 \lambda d_{\mathrm{px}}}{2 M \mathrm{NA}} \sqrt{\frac{\pi}{N_{\mathrm{a}} N_{\mathrm{fw}} \nu}} .
$$

For the green channel of Fig. 2, we have $N_{\mathrm{fw}}=4 \times 10^{4}$, $N_{\mathrm{a}}=36, M=60, d_{\mathrm{px}}=5.7 \mu \mathrm{m}, \mathrm{NA}=0.95, \lambda=0.53 \mu \mathrm{m}$, and $\nu=1 / 2$, yielding $\hat{\sigma}_{\text {noise }}=589 \mathrm{~nm}^{2}$, in agreement with the measured noise. The blue and red channels have a factor of $\sqrt{2}$ larger noise due to the smaller $\nu$. This detection limit could be improved by using an oil immersion objective with $1.45 \mathrm{NA}, M=150$, and $N_{\mathrm{a}}=1800$ possible in a $60 \mathrm{~s}$ video, for which Eq. (1) yields $\hat{\sigma}_{\text {noise }}=43 \mathrm{~nm}^{2}$, which would allow measuring single GNPs down to $10 \mathrm{~nm}$ diameter.

We note that the finite angular range of the objective implies that it collects also a fraction of the scattered light, leading to an underestimate of the extinction. The solid angle in the sample with 1.5 refractive index collected by the objective is $1.6 \mathrm{sr}$, which for isotropic scattering is $13 \%$ of the scattered light. We could correct for this by adding $13 \%$ of the measured $\sigma_{\text {sca }}$ to $\sigma_{\text {ext }}$. We also note that $\sigma_{\text {sca }}$ is determined using the scattering of the dark-field excitation into the objective, which also has a certain angular range that needs to be considered if the scattering is sufficiently anisotropic.

Furthermore, we measured the dependence of $\sigma_{\text {ext }}$ on the linear polarization angle $\theta$ of the excitation light, which is a sensitive probe of NP asphericity, by inserting a linear polariser in the illumination beam path before the condenser. The resulting $\sigma_{\text {ext }}(\theta)$ is shown in Fig. 4 (right) for $\theta$ between $0^{\circ}$ and $180^{\circ}$ in steps of $10^{\circ}$ for two selected GNPs in the red channel. We analyze these results by fitting the expression $\sigma_{\text {ext }}(\theta)=\sigma_{0}\left(1+\alpha \cos \left(2\left(\theta-\theta_{0}\right)\right)\right)$, where $\sigma_{0}$ is the polarization-averaged $\sigma_{\text {ext }}, \alpha \geq 0$ is the amplitude of the polarization dependence, and $0 \geq \theta_{0} \geq \pi$ is an angular offset, indicating the direction of the NP asymmetry. To estimate the influence of the measurement noise on the fit parameters, we calculated their distribution over Gaussian random fluctuations of the fitted $\sigma_{\text {ext }}(\theta)$ with a standard deviation $\hat{\sigma}_{\text {noise }}$ GNP1 has a fitted $\alpha=0.07$, and its distribution has a mean value $\bar{\alpha}=0.15$ and a standard deviation 


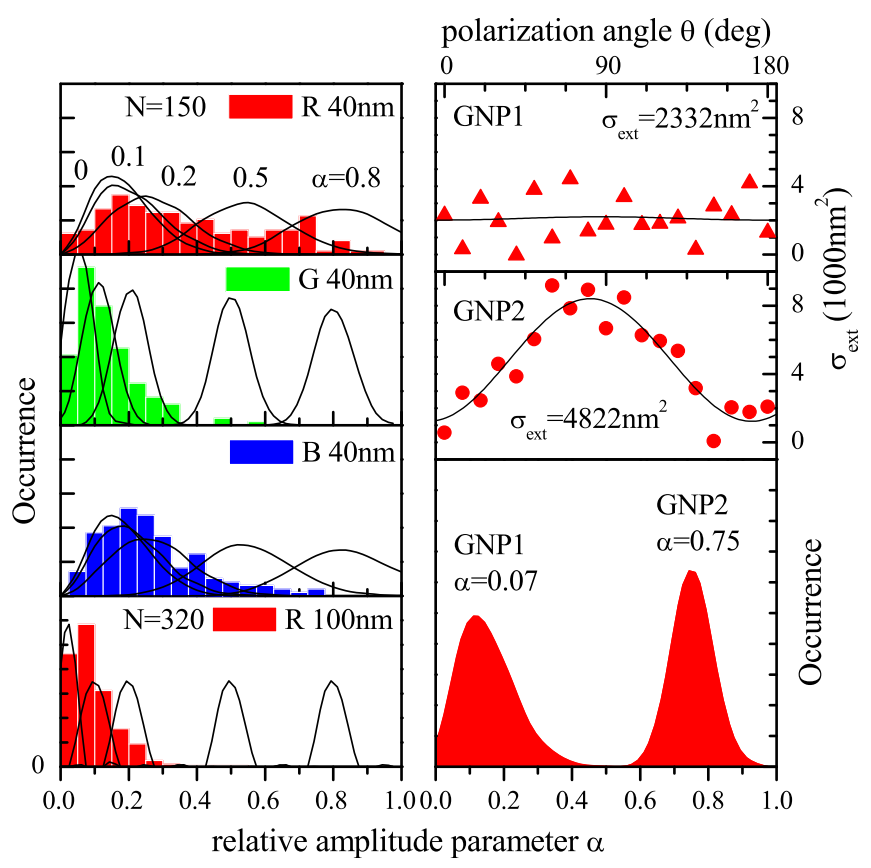

FIG. 4. Left column: Distributions of the amplitude $\alpha$ for 150 GNPs of $40 \mathrm{~nm}$ diameter in the R, G, and B color channels, and 320 GNPs of $100 \mathrm{~nm}$ diameter in the $\mathrm{R}$ channel. The distributions due to $\hat{\sigma}_{\text {noise }}$ for GNPs of $\alpha=0,0.1$, $0.2,0.5,0.8$ are given as black lines using $\bar{\sigma}_{\text {ext }}$ as given in Fig. 2. Right column: $\sigma_{\text {ext }}(\theta)$ in the R channel with fits for two $40 \mathrm{~nm}$ GNPs, and distribution of the deduced amplitude parameter $\alpha$ by the measurement noise $\hat{\sigma}_{\text {noise }}$.

$\hat{\alpha}=0.08$. GNP2 instead is significantly non-spherical with a fitted $\alpha=0.75$ and a distribution with $\bar{\alpha}=0.75$ well above $\hat{\alpha}=0.06$. The red channel is used here, as it is most sensitive to LSPR shifts due to asphericity.

The distribution of $\alpha$ over the NP ensemble is shown in Fig. 4 (left) for $40 \mathrm{~nm}$ and $100 \mathrm{~nm}$ GNPs for different color channels. For comparison, the simulated distribution of $\alpha$ for GNPs having $\sigma_{\text {ext }}(\theta)$ given by the fit function is shown for $\alpha=0,0.1,0.2,0.5,0.8$ in Fig. 4 as black lines using $\sigma_{0}=\bar{\sigma}_{\text {ext }}$ of the color channel. The comparison shows that the polarization dependence can identify non-spherical GNPs through the distinct values of $\alpha$. To further infer the NP geometrical aspect ratio from these data, a comparison with theory is needed which will be reported in a forthcoming work.
In conclusion, we have shown that conventional widefield microscopy can be implemented with a consumer camera to extract quantitative values of polarization-resolved extinction, scattering, and absorption cross-sections of individual nanoparticles and generate histograms for statistical characterization of large numbers of particles. Although quasi-spherical gold nanoparticles of $40 \mathrm{~nm}$ and $100 \mathrm{~nm}$ diameter were used in this work for proof of principle, the technique is applicable to any nanoparticles, with a detection sensitivity limit in the order of $100 \mathrm{~nm}^{2}$. Importantly, this technique can be adopted by any laboratory equipped with conventional wide-field microscopy as a tool to quantify the linear optical response of a statistically significant number of individual nanoparticles.

This work was supported by the UK EPSRC Research Council under the Leadership fellowship award of P.B., Grant Nos. EP/I005072/1 and EP/I016260/1. The analysis program used is available as IMAGEJ plug-in at http://langsrv. astro.cf.ac.uk/Crosssection.

${ }^{1}$ W. Barnes, A. Deraux, and T. Ebbesen, Nature 424, 824 (2003)

${ }^{2}$ K. Awazu, M. Fujimaki, C. Rockstuhl, J. Tominaga, H. Murakami, Y. Ohki, N. Yoshida, and T. Watanabe, J. Am. Chem. Soc. 130, 1676 (2008).

${ }^{3}$ H. Atwater and A. Polman, Nat. Mater. 9, 205 (2010).

${ }^{4}$ L. Cognet, S. Berciaud, D. Lasne, and B. Lounis, Anal. Chem. 80, 2288 (2008).

${ }^{5}$ F. Masia, W. Langbein, P. Watson, and P. Borri, Opt. Lett. 34, 1816 (2009).

${ }^{6}$ K. Kneipp, H. Kneipp, I. Itzkan, R. Dasari, and M. Feld, J. Phys.: Condens. Matter 14, R597 (2002).

${ }^{7}$ A. McFarland and R. Van Duyne, Nano Lett. 3, 1057 (2003).

${ }^{8}$ C. Sonnichsen, B. Reinhard, J. Liphardt, and A. Alivisatos, Nat. Biotechnol. 23, 741 (2005).

${ }^{9}$ L. Anderson, K. Mayer, R. Fraleigh, Y. Yang, S. Lee, and J. Hafner, J. Phys. Chem. C 114, 11127 (2010).

${ }^{10}$ A. Tcherniak, J. Ha, S. Dominguez-Medina, L. Slaughter, and S. Link, Nano Lett. 10, 1398 (2010).

${ }^{11}$ O. Muskens, P. Billaud, M. Broyer, N. Del Fatti, and F. Vallée, Phys. Rev. B 78, 205410 (2008).

${ }^{12}$ M. van Dijk, A. Tchebotareva, M. Orrit, M. Lippitz, S. Berciaud, D. Lasne, L. Cognet, and B. Lounis, Phys. Chem. Chem. Phys. 8, 3486 (2006).

${ }^{13}$ C. Noguez, Opt. Mater. 27, 1204 (2005).

${ }^{14}$ M. van Dijk, "Nonlinear-optical studies of single gold nanoparticles," Ph.D. dissertation (Universiteit Leiden, 2007).

${ }^{15}$ G. Mie, Ann. Phys. 330, 377 (1908). 